CASE REPORT

\title{
Challenges in The Management of Toxic Multi Nodular Thyroid in Pregnancy who had Crisis Thyroid
}

\author{
Kurnia Alisaputri ${ }^{1}$, Sony Wibisono ${ }^{2 *}$ (D) \\ ${ }^{1}$ Department of Internal Medicine, Faculty of Medicine, Universitas Airlangga - Dr. Soetomo General \\ Academic Hospital, Surabaya, Indonesia
}

${ }^{2}$ Division of Endocrin and Metabolic Diabetes, Department of Internal Medicine, Faculty of Medicine - Dr. Soetomo General

Academic Hospital, Surabaya, Indonesia

\begin{abstract}
A B S T R A C T
Managing crisis thyroid in toxic multi nodular thyroid in pregnancy is challenging. The challenges are controlling thyroid hormone in mother without affecting development of fetus. This case objective is to highlight comprehensive treatment going to avoid maternal \& infant mortality. Actually, crisis thyroid in pregnancy is a preventable death. An upgrading resources team in peripheral public health service is necessary. A woman, 43 years old in 13th week of her third pregnancy, admitted in emergency room with palpitating and vomiting as her chief complaints. Her complaints had started since she stopped taking propylthiouracyl. She used to take it since diagnosed hyperthyroid. She was diagnosed crisis hyperthyroid in pregnancy. The first main goal was to stabilize the patient, curing the precipitate factors, and assist her to have a healthy baby. She was discharged from hospital day 7th. She was routinely checked FT4 for evaluation and switch on Thyrozol during 25-26th weeks and continued it until postpartum. Her baby was delivered vaginally with normal APGAR score and growing well. Based on her experience, she agreed to have IUD to prevent unplanned pregnancy.
\end{abstract}

Keywords: Crisis thyroid, Hyperthyroid in pregnancy, Toxic multinodular thyroid, Maternal mortality, Infant mortality, Preventable death, Public health

Correspondence: Sony Wibisono

E-mail: sony.wibisono@fk.unair.ac.id

Article history: • Received 3 January 2021 • Received in revised form 2 February $2021 \bullet$ Accepted 3 March 2021

\section{INTRODUCTION}

Toxic Multinodular Thyroid is one causal of crisis thyroid. In Pregnancy, it can be overlapping with hyperemesis gravidarum and Grave's disease. Toxic multi nodular goiter (MNG) becomes second causal of thyrotoxicosis. It is unremitting and develops slowly, with more subtle than Grave's. It induces a hyperthyroid since the functional capacity of the thyroid cells then become independent of regulation by thyroid-stimulating hormone (TSH) focal and/or diffuse hyperplasia of thyroid follicular cells occurs, a long phase of subclinical hyperthyroidism can precede the appearance of overt symptoms (Fulara and Fulara, 2017). MNG are characterized by systemic symptom such as agitation to psychosis, warm skin, tachycardia as sign of increasing cardiac output, $60 \%$ hyperthyroid patient has atrial fibrillation, dyspnea, hyper defecation, malabsorption, abnormality bone composition, and polyuria (Ross, 2018).

\section{CASE REPORT}

In July 2017, a woman, 43 years old in 13th week of her third pregnancy, admitted in emergency room with palpitating and vomiting as her chief complaints. She had nausea and vomiting for a month. Nausea and vomiting didn't come with headache. She drooled more than usual then it was followed with nausea and vomiting. She also had fever, palpitation, and shortness of breath, stomachache, and dizziness. Her complaints had started since she stopped taking propylthiouracyl (PTU) which she used to take it regularly since diagnosed hyperthyroid 2 years ago. She had stopped taking PTU since her nausea was getting worse. This complaints appeared at the same time with absent of menstrual period and her breast became tender this last 3 months. Now, she urinates more often these recent days.

In 2015, Pts was diagnosed with hyperthyroid then given PTU and Propanolol. She visited a Midwife then stopped her Depo Medroxy Progesterone Acetate (DMPA) as her contraception at that time without replacing with other contraception. She had twice previous premature birth, her first son is 25 years old and the younger is 23 years old now. Her last menstruation was in April 2017 and had positive result for pregnancy test. In June 2017, she had hyperemesis then stopped taking PTU.

The patient was in weak condition, agitated, with blood pressure of $160 / 100 \mathrm{mmHg}$, heart rate was 130 beat per minute (bpm) regular, respiration rate 27 times per minute (tpm), body temperature 37.6 degree celcius. From head and neck examination we found enlargement of thyroid glands 
and no exophthalmos with increase of jugular venous pressure. On heart examination we found strong heart pulsation of ictus cordis in mid clavicular line. Single S1 and $\mathrm{S} 2$, with gallop, but without murmur nor extra systole. From the extremities we found no myxedema, but with wet and cold palm. Total of Burch - Wartofsky (BW) score was 55. Body weight was $51 \mathrm{~kg}$, body height was $155 \mathrm{~cm}$, BMI was 21 (normal).

We performed several examination to establish diagnosis and to find out her future management strategies. The admission of thyroid function test were Thyroid Stimulating Hormone (TSH) is $0.001(0.55-4.78 \mathrm{IU} / \mathrm{ml})$, Free Thyroid-4 (FT4) is $19.51 \quad(0.89-1.76 \mathrm{mg} / \mathrm{dL})$. From obstetric ultrasonography, the gestational age was equal to $14 / 15$ weeks, singleton, with fundal placenta, normal amount of amniotic fluid with normal fetal movement and was concluded that there are no suspicion of major congenital anomaly. She was advised to have ultrasonography examination repeated next month. ECG showed sinus tachycardia 130 beat per minute.

Both physical and laboratory examination results indicate that the patient is inflicted with Toxic Multinodular Thyroid in pregnancy, a particularly high risk case and demanding a well management to safe patient and her baby's life.
The first main goal was to stabilize the patient, curing the precipitate factors in order to treat the thyroid crisis. We managed patient using guideline crisis thyroid in pregnancy of Association Thyroid of America (ATA). Patient should be in Intensive Care setting. First of all we gave oxygenation to the patient with reservoir mask 8 to 10 liter per minute (lpm), with normal saline infusion $500 \mathrm{cc}$ in 6 hours. We gave high calorie and high protein diet of $1800 \mathrm{kcal}$. Hyperthyroid medication started with therapy for thyroid crisis. Patient should take PTU tablet every 4 hours, it was started by 400 $\mathrm{mg}$ of loading dose, then followed by maintaining dose PTU $100 \mathrm{mg}$ every 4 hours, orally. She was also given Iodine drops, methylprednisolone, symptomatic therapy in order to relieve her condition. Her baby was regularly evaluated by obstetric team. Vital sign monitoring and complaints must be taken as priority. On second day, her condition was well improved with BW score 40 without vomiting. On seventh day, the result of TSH was 0,008 (0.55-4.78 IU/ml), and FT4 was $1,75(0.89-1.76 \mathrm{mg} / \mathrm{dL})$. So, we diagnosed her with subclinical hyperthyroid. She was planned to continue PTU $100 \mathrm{mg}$, Propranolol $10 \mathrm{mg}$, and ASA $80 \mathrm{mg}$, all once daily and also MP $16 \mathrm{mg}$ three times a day orally. The PTU had must be taken until 1st semester, Propranolol had must be taken 3 days after discharge only, regarding its effect on pregnancy.

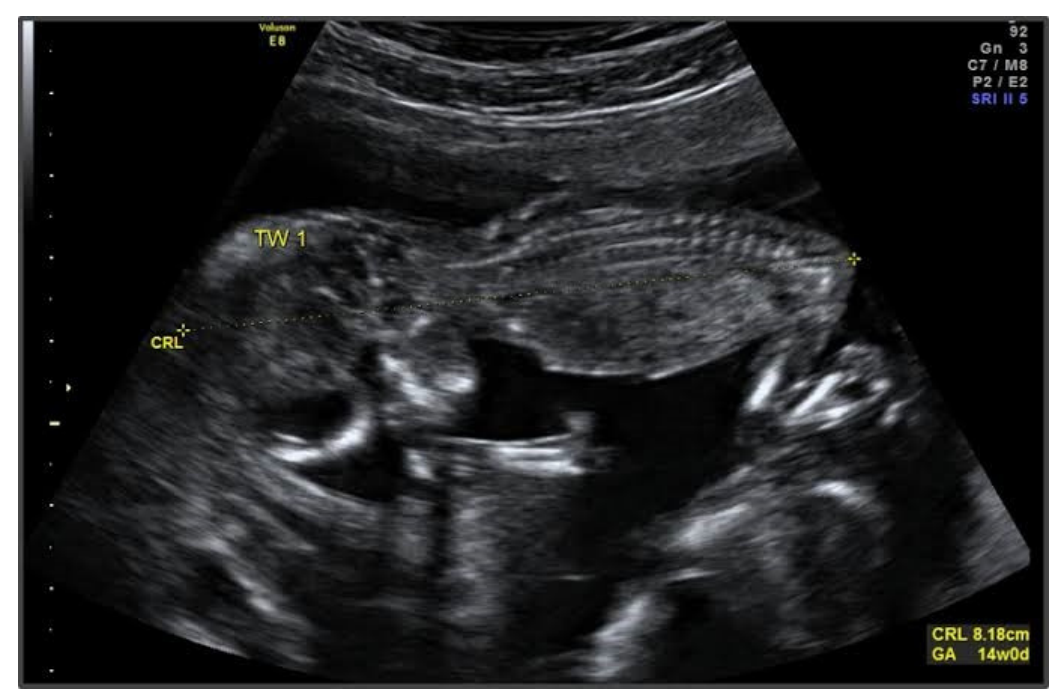

Figure 1. Obstetric USG of patient on 14-15 weeks old

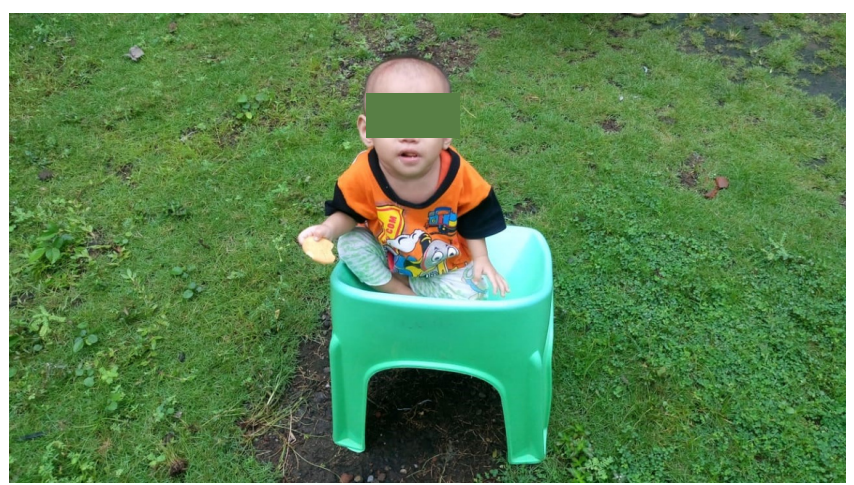

Figure 2. A 6 month old baby boy 


\section{DISCUSSION}

Overt hyperthyroidism (low thyroid-stimulating hormone (TSH), elevated free thyroxine (T4) and/or triiodothyronine (T3) is relatively uncommon during pregnancy, occurring in 0.1 to 0.4 percent of all pregnancies (Krassas et al., 2016). About $1-2 \%$ of hyperthyroidism has thyroid crisis during pregnancy (Ma et al., 2018). The most frequent causal of hyperthyroidism are Grave's disease, toxic adenoma, and toxic multinodular goiter (Ross, 2018). Genetically, Asian women are more likely to have gestational thyrotoxicosis and hyperemesis gravidarum due to raising in production and metabolism of human-chorionic gonadotropin (hCG) (Purnamasari et al., 2013).

Toxic multi nodular goiter (MNG) becomes second causal of thyrotoxicosis. It is unremitting and develops slowly, with more subtle than Grave's (Fulara and Fulara, 2017). Its characterized by systemic symptom such as agitation to psychosis, warm skin, tachycardia as sign of increasing cardiac output, $60 \%$ hyperthyroid patient has atrial fibrillation, dyspnea, hyper defecation, malabsorption, abnormality bone composition, and polyuria (Ross, 2018). Toxic or hot nodules secrete THs independent of the pituitary because this tissue contains mutated TSHRs. Thyroxine levels typically are elevated in these patients sometimes only T3 levels are increased. Consequently if T4 concentrations are normal in such patients T3 levels should be determined to rule out T3 toxicosis. In toxic multinodular goiter, the thyroid gland normally enlarges in reaction to an increased demand for THs that occurs in pregnancy, iodine deficiency and immunologic, genetic disorders. Autonomous follicles may produce excessive $\mathrm{TH}$ unregulated by $\mathrm{TSH}$, resulting in thyrotoxicosis symptoms similar to GD without infiltrative ocular manifestations or myxedema (Alemu et al., 2016).

In laboratory findings, measurement of TSH level is the only initial test necessary in a patient with a possible diagnosis of hyperthyroidism without evidence of pituitary disease. If the TSH level is low, then FT4 should be measured to evaluate for thyrotoxicosis. Measurement of FT3 is helpful in the clinical diagnosis of thyrotoxicosis when the FT4 values are unexpectedly normal (Alemu et al., 2016). Women with FT3, FT4 above the reference range along with TSH value $<0.1 \mathrm{mIU} / \mathrm{L}$ were classified as having overt hyperthyroidism while those having FT3, FT4 in normal range with $\mathrm{TSH}<0.1 \mathrm{mIU} / \mathrm{L}$ were diagnosed as having subclinical hyperthyroidism. Normal range for TPO antibody was $<35 \mathrm{IU} / \mathrm{mL}$ and value greater than or equal to indicate elevated Anti-TPO in serum (Rajput et al., 2015).

In patient with hyperthyroid and stop menstruation period we have to think about differential diagnoses such as gestational trophoblastic disease and gestational hyperthyroid. In the past, approximately 55 to 60 percent of women with trophoblastic disease had clinically evident hyperthyroidism at the time of diagnosis, which could be severe. However, in a review of 196 patients from the United Kingdom treated for gestational trophoblastic disease between 2005 and 2010, biochemical hyperthyroidism was present in 7 percent and clinical hyperthyroidism in only 2 percent (Walkington et al., 2011).

Pregnancy poses to maternal thyroid gland as hormone requirements are increased during gestation. Beta HCG are homolog of $\mathrm{TSH}$, so it seats on $\mathrm{TSH}$ receptors to stimulate thyroid hormones. In the other hand, there is also an estrogen mediated increased in circulating of thyroid - binding globulin (TBG) 2-3 times in serum during pregnancy and increase TBG production in liver. Enhance, TBG is one of numerous protein that transport thyroid hormone in the blood with high affinity for thyroxin (T4) in serum a few weeks after conception and ranges a plateau during mid-gestational period. Both mechanism between estrogen mediated and hepatic synthesis of TBG lead to increases the half-life from 15 minutes to 3 days to fully sialylated TBG. Elevated levels of TBG lead to lowered free T4 concentrations which results in increased TSH secretion by pituitary and automatically enhanced production and thyroid hormone secretion (Alemu et al., 2016).

Treatment for thyroid storm or heart failure is similar and should be carried out in an intensive care area that may include special-care units within labor and delivery. An hour or two after initial thionamide administration, iodide is given to inhibit thyroidal release of T3 and T4. It can be given intravenously as sodium iodide or orally as either saturated solution of potassium iodide (SSKI) or Lugol solution. With a history of iodine-induced anaphylaxis, lithium carbonate, $300 \mathrm{mg}$ every 6 hours, is given instead. Most authorities recommend dexamethasone $2 \mathrm{mg}$ intravenously every 6 hours for four doses, to further block peripheral conversion of T4 to T3. If a $\beta$-blocker drug is given to control tachycardia, its effect on heart failure must be considered. Propranolol, labetalol, and esmolol have all been used successfully. Coexisting severe preeclampsia, infection, or anemia should be aggressively managed before delivery is considered (Cunningham, 2014). Thyrotoxicosis during pregnancy can nearly always be controlled by thionamide drugs. Propylthiouracil (PTU) has been historically preferred because it partially inhibits the conversion of T4 to T3 and crosses the placentaless readily than methimazole. The initial thionamide dose is empirical. For non-pregnant patients, the American Thyroid Association recommends that methimazole be used at an initial higher daily dose of 10 to $20 \mathrm{mg}$ orally followed by a lower maintenance dose of 5 to $10 \mathrm{mg}$. If PTU is selected, a dose of 50 to $150 \mathrm{mg}$ orally three times daily may be initiated depending on clinical severity. At Parkland Hospital, it is initially given for 300 or $450 \mathrm{mg}$ daily in three divided doses for pregnant women. 
Occasionally, daily doses of $600 \mathrm{mg}$ are necessary. As discussed, they do not transition women to methimazole during the second trimester. The goal is treatment with the lowest possible thionamide dose to maintain thyroid hormone levels slightly above or in the high normal range while TSH levels remains suppressed (Fulara and Fulara, 2017). Serum free T4 concentrations are measured every 4 to 6 weeks (Cunningham, 2014).

All pregnant women should consume approximately $250 \mu \mathrm{g}$ iodine daily. In order to achieve a total of $250 \mu \mathrm{g}$ iodine ingestion daily, strategies may need to be varied based on country of origin (Alemu et al., 2016).

Given PTU hepatotoxicity concerns, experts currently recommend using low-to-moderate MMI doses as a first-line therapy in lactating mothers. PTU should be reserved only as a second-line agent for cases of severe hyperthyroidism especially thyroid storm and allergic reactions to previous MMI treatment. ATD should be administered in divided doses immediately following each feeding. Evaluation of thyroid function tests is advisable at least 3-4 weeks after the initiation of breastfeeding (Hudzik and ZubelexiczSzkodzinska, 2016).

\section{CONCLUSION}

A 43 year old woman whom diagnosed thyroid crisis with comorbid gravida, had been treated with PTU, lugol, and metilprednisolon. Since her pregnancy during first trimester, therapy of methimazole was not the first choice. She was discharge with sub clinical hyperthyroid then routinely had her thyroid condition and pregnancy checked up in local area. The therapy target was to keep her in subclinical hyperthyroid. She was routinely checked FT4 for regular evaluation and also under supervision of obstetrician. Combination of well pregnancy program and obvious care treatment in hyperthyroid will safe mother and child.

\section{REFERENCES}

Alemu A, et al. 2016. Thyroid hormone dysfunction during pregnancy: a review. Int J Reprod Biomed 14(11):677-686.

Alexander EK, et al. 2017. 2017 Guidelines of the American thyroid association for the diagnosis and management of thyroid disease during pregnancy and the postpartum. Thyroid 27(3):315-389. doi: 10.1089/thy.2016.0457.

Cunningham FG. 2014. Williams Obstetric 24th edition. New York, McGraw-Hill.

Fulara SN and Fulara NY. 2017. Toxic multinodular goitre (thyrotoxicosis with hyperthyroidism) induced cardiomyopathy: a Case Report. Open Journal of Clinical Diagnostics 07(02):67-72. doi: 10.4236/ojcd.2017.72007.

Hudzik B and Zubelewicz-Szkodzinska B. 2016. Antithyroid drugs during breastfeeding. Clinical Endocrinology 85(6):827-830. doi: 10.1111/cen.13176.

Krassas GE, Poppe K, Glinoer D. 2010. Thyroid function and human reproductive health. Endocrine Reviews 31(5):702755. doi: 10.1210/er.2009-0041.

Ma Y, et al. 2018. Impending thyroid storm in a pregnant woman with undiagnosed hyperthyroidism: a case report and literature review. Medicine 97(3):e9606. doi: 10.1097/ MD.0000000000009606.

Purnamasari D, et al. 2013. Indonesian clinical practice guidelines for the management of thyroid dysfunction during pregnancy. Journal of the ASEAN Federation of Endocrine Societies 28(1):18-20. doi: 10.15605/jafes.028.01.04.

Rajput R, et al. 2015. Prevalence of thyroid dysfunction among women during the first trimester of pregnancy at a tertiary care hospital in Haryana. Indian Journal of Endocrinology and Metabolism 19(3):416. doi: 10.4103/ 2230-8210.152791.

Ross DS. 2018. Overview of the clinical manifestations of hyperthyroidism in adults, UpToDate. Available from https://www.uptodate.com/contents/overview-of-the-clinicalmanifestations-of-hyperthyroidism-in-adults. Accessed 11 September 2020).

Walkington L, et al. 2011. Hyperthyroidism and human chorionic gonadotrophin production in gestational trophoblastic disease. British Journal of Cancer 104(11):1665-1669. doi: 10.1038/bjc.2011.139. 